\title{
Questes
}

vestes Revue pluridisciplinaire d'études médiévales

$7 \mid 2004$

Les tabous

\section{Ce con (ne dira pas)}

\section{Mathilde Grodet}

\section{(2) OpenEdition}

\section{Journals}

\section{Édition électronique}

URL : http://journals.openedition.org/questes/2790

DOI : 10.4000 /questes. 2790

ISSN : 2109-9472

\section{Éditeur}

Les Amis de Questes

\section{Édition imprimée}

Date de publication : 15 juin 2004

Pagination : 30-35

ISSN : 2102-7188

\section{Référence électronique}

Mathilde Grodet, « CE CON (NE DIRA PAS)», Questes [En ligne], 7 | 2004, mis en ligne le 15 janvier 2014, consulté le 22 septembre 2020. URL : http://journals.openedition.org/questes/2790 ; DOI : https:// doi.org/10.4000/questes. 2790 
Le moine ne peut donc s'alimenter que de la chair sacrée, c'est-à-dire du corps du Christ et par là même du sang du Christ. La seule viande qui peut être avalée par le moine est « la viande espirituelle» :

«Elle est la vitaille de l'ame qui tien le cuer fort et seur en chastel encontre les ennemis est la parole de Dieu, c'est assavoir souvent et voulentiers oÿr les sermons et la parole de Dieu et de edificacion. (...) Une autre viande moult precieuse pour l'ame et le cuer bien garder en vie de grace. (...) La tierce viande, qui est la plus noble et la plus haulte, si est souvent communier et recevoir le precieux corps Nostre Seigneur Jhesu Crist ». «Ceste viande esperituelle nourrist l'ame en grace et la fait croistre en vertu et en l'amour de Dieu. (...) tout aussi convient il l'ame souvent repaistre de sa viande, c'est du corps Nostre Seigneur, a ce qu'elle puisse croistre et estre confermee en la grace et en l'amour de Dieu » ${ }^{x x v i}$.

L'ascèse du corps, le jeûne et l'abstinence leur permettent ainsi de lutter contre les vices et de mener une vie spirituelle parfaite. La viande représente un tabou alimentaire pour le monde monastique médiéval car elle est non seulement l'aliment «qui ne peut être mangé », mais plus encore le symbole d'un interdit motivé ici par son caractère impur.

\section{Ce con (ne dira pas) $)^{x x v i i}$}

\section{Mathilde GRODET}

Lors d'une de nos réunions, nous nous sommes demandé si le sexe de la femme constituait un tabou littéraire au Moyen Age. Pour répondre à cette question, j'ai observé dix-neuf récits érotiques ${ }^{\mathrm{xxviii}}$, oeuvres qui, affirmant une thématique sexuelle, me paraissaient avoir le moins de pudeur à parler du sexe féminin. Or 
leur lecture peut surprendre puisque celui-ci, bien que présent, est très peu mentionné. Cette constatation soulève deux nouvelles interrogations : quelles sont les stratégies d'évitement utilisées par les auteurs pour ne pas avoir à désigner ce sexe ? Et surtout où se situe l'interdit?

A la lecture de ces récits, nous remarquons en effet une sous-représentation du sexe féminin à la fois quantitative et qualitative. Les termes désignant le sexe de la femme sont nettement moins fréquents que ceux désignant celui de l'homme. Alors que les mots vit, couilles, couillons sont présents dans pratiquement tous nos textes, on ne relève le terme con que dans six de nos récits. Pourtant les situations mises en scène ne justifient pas un tel écart: il n'y a ni scène d'homosexualité masculine ${ }^{\mathrm{xxix}}$, ni scène de masturbation par exemple. Autre fait significatif, les descriptions de l'objet sont exceptionnelles. Ici encore la comparaison avec le sexe de l'homme montre une différence nette. Les vits peuvent être gros et carrés, grands, raides, pendouillants... Mais le con est rarement décrit et quand il l'est, c'est toujours de façon imagée. Nous observons donc une certaine réticence à nommer le sexe de la femme pourtant très sollicité dans ces textes.

Si nous cherchons à savoir quelles sont les ruses des auteurs pour éviter de désigner et de décrire ce sexe, nous nous apercevons que leurs stratégies vont de l'évitement total à son évocation voilée. Ainsi certains auteurs privilégient des situations telles qu'on ne peut pas voir le sexe de la femme. Celui-ci est caché 
par un vêtement, comme dans le fabliau Cele qui fu foutue et desfoutue por une Grue où le jeune homme doit chercher soz [la] pelice et solieve la chemise. Il peut être dissimulé également par l'homme qui est toujours sur sa partenaire. $L e$ Vilain de Bailluel aperçoit ainsi le capuchon du prêtre mais pas sa femme. D'autres écrivains tirent parti de l'obscurité de la nuit. Gombert et les deus Clers et Trubert sont des exemples intéressants puisque l'obscurité totale y est à l'origine de duperie et de quiproquo. Quand il n'est pas caché, le corps de la femme peut être considéré comme un tout et l'auteur évoque le coït sans s'arrêter aux parties génitales. C'est là le cas le plus fréquent, mais il arrive aussi que l'on assiste à un glissement vers d'autres parties du corps évocatrices, notamment le cul, les rains, les hanches, mais aussi le paignil attesté une seule fois dans La Damoiselle qui ne pooit ö̈r parler de foutre. Enfin les figures de style permettent aux auteurs de respecter ce qui semble un tabou et de montrer toute leur habileté. La plus évidente est la périphrase. Citons : la o lo mal as dames tient extraite de La Damoiselle qui sonjoit et ce passage de Cele qui fu foutue et desfoutue por une Grue :

La demoisele giete jus et entre les janbes li entre si li enbat lou foutre el ventre.

La métaphore est plus intéressante car elle offre la possibilité d'un développement long et donc d'une description. Dans La saineresse, une femme raconte à son mari la relation qu'elle vient d'avoir avec un autre homme en filant une métaphore médicale. Mais c'est surtout dans La Damoiselle qui ne 
pooit oïr parler de foutre que l'emploi de la métaphore est le plus abouti. Le sexe de la femme devient un véritable paysage bucolique et l'auteur n'omet aucune de ses parties. Le calembour est également présent dans ces récits. Il est même à l'origine du titre du fabliau Guillaume au faucon. Signalons pour finir l'emploi du prénom Golïas, dont nous n'avons pu déterminer la source, pour désigner la vulve. Les stratagèmes, nous le voyons, sont nombreux. Il y a tout un jeu littéraire de la part des auteurs pour ne pas parler du sexe de la femme ou pour en parler sans jamais le dire.

Pourtant toutes ces précautions peuvent paraître paradoxales dans des récits dont l'intrigue est ouvertement sexuelle et qui affichent une certaine liberté de ton. Il y a vraisemblablement un tabou, reste à savoir ce à quoi il correspond exactement. Deux pistes s'ouvrent à nous. D'abord l'impossibilité de décrire le sexe de la femme n'est pas liée à l'objet en lui-même qui est utilisé, touché et même écouté (Le Chevalier qui fist parler les cons), mais à la vue de l'objet. Nous avons déjà remarqué que le sexe de la femme est souvent dissimulé. En même temps les nombreuses scènes de voyeurisme témoignent de la fascination qu'il provoque et du vif désir de l'apercevoir. Cependant seuls trois récits mettent en scène des personnages qui regardent effectivement le sexe d'une femme. Parmi eux Trubert doit être mis à part, car ce sont des jeunes filles encore impubères que l'homme observe : il voit les connez bufiz, sanz barbe, pouvons-nous lire. Dans les deux autres textes La Dame escoilliee et Berangier 
au lonc cul, nous remarquons un fait étrange : quand un personnage regarde le sexe d'une femme, il voit en fait le sexe d'un homme! Dans La Dame escoilliee, un gendre agacé par l'attitude dominatrice de sa belle mère envers son mari fait semblant de l'émasculer. Mais quand il présente les testicules (de taureau en réalité) aux yeux de la femme et de son époux, aucun des deux ne doutent qu'ils appartiennent en effet à la dame. L'héroïne de Berangier au lonc cul, déguisée en chevalier, défie son mari poltron et lui laisse une alternative : ou il se bat ou il lui baise les fesses. Mais lorsqu'il s'approche pour le faire

$$
\begin{aligned}
& \text { [...] cil regarde la crevace } \\
& \text { du cul et du con : ce li sanble } \\
& \text { que trestot li tenist ensanble. } \\
& \text { A lui meïsme pense et dit } \\
& \text { que onques si lonc cul ne vit. }
\end{aligned}
$$

Seul le sexe des femmes impubères ou viriles par leur comportement ou leurs vêtements semble pouvoir être regardé par les hommes. L'interdit touche ici la vision du sexe, or on ne peut décrire ce que l'on ne peut pas voir. A cette impossibilité de décrire s'ajoute la difficulté de dire qui nous permet de nous interroger sur le lien entre la verbalisation et le tabou. La Damoiselle qui ne pooit oïr parler de foutre est très clair à ce sujet. Ce fabliau met en scène une jeune fille qui ne montre aucune répugnance à faire l'amour, mais ne supporte pas les mots qui évoquent l'acte sexuel, d'où la longue métaphore dont nous avons parlé. D'autres récits soulignent le fait que le tabou réside non dans l'acte mais dans sa verbalisation. Dans La saineresse, le sommet de la transgression et ce qui fait le plus plaisir à la bourgeoise, ce n'est pas de tromper son mari, mais 
de le lui raconter en utilisant un double langage qu'il ne comprend pas. Il nous faut citer enfin le Lai del lecheor. Il nous raconte qu'une fois par an une assemblée se réunissait; chacun rapportait les aventures extraordinaires qu'il avait vécues; on faisait un lai du meilleur récit et on lui donnait pour titre le nom du héros. Or il advint qu'une dame fit remarquer que la source de tous ces hauts faits n'était rien d'autre que le con des dames et qu'il méritait bien un lai. Mais dans ce texte, l'auteur retarde au maximum l'apparition du mot qui crée la surprise dans la bouche de la dame. En outre il refuse de le prendre à son compte :

D'icest lai dient li plusor que c'est le lai du Lechëor ; $\mathrm{Ne}$ voil pas dire le droit non c'on nu me tort a mesprison.

Il semblerait donc que la faible fréquence des termes évoquant la vulve ne soit pas le résultat d'une pudeur, mais bien du respect d'un tabou lié au langage.

Bien sûr cette étude est trop rapide. Il faudrait élargir le corpus et mieux mesurer la place du sexe de la femme dans l'imaginaire médiéval au regard des travaux d'historiens notamment. Elle nous permet toutefois d'approcher l'essence d'un tabou littéraire, interdiction de voir, mais surtout de dire. Contrainte pour les auteurs, son respect révèle aussi leur adresse. 